\title{
CRESCIMENTO E SINTOMAS EM MUDAS DE ESPINHEIRA-SANTA COM OMISSÃO DE NITROGÊNIO, FÓSFORO E POTÁSSIO
}

\author{
Eliziane Luiza Benedetti ${ }^{1}$, Charlote Wink ${ }^{2}$, Delmar $\operatorname{Santin}^{3}$, Fernanda Sereda ${ }^{4}$, Luis Fernando Roveda ${ }^{5}$, \\ Beatriz Monte Serrat ${ }^{6}$
}

${ }^{1}$ Enga Agrônoma, Doutoranda em Solos e Nutrição de Plantas, UFV, Viçosa, MG, Brasil - elibettiagro@ yahoo.com.br ${ }^{2}$ Eng $^{\mathrm{a}}$ Florestal, Doutoranda em Engenharia Florestal, UFSM, Santa Maria, RS, Brasil - charlotewink@ yahoo.com.br ${ }^{3}$ Eng. Florestal, Doutorando em Solos e Nutrição de Plantas, UFV, Viçosa, MG, Brasil - desantinflorestal@ yahoo.com.br ${ }^{4}$ Eng $^{a}$ Agrônoma, M.Sc., Estre Ambiental, Curitiba, PR, Brasil - fernanda.sereda @estre.com.br

${ }^{5}$ Eng. Agrônomo, Doutorando em Produção Vegetal, UFPR, Curitiba, PR, Brasil - lfroveda@uol.com.br ${ }^{6}$ Enga $^{\mathrm{a}}$ Agrônoma, Dr ${ }^{\mathrm{a}}$., Depto. Solos e Engenharia Agrícola, UFPR, Curitiba, PR, Brasil - bmserrat@ yahoo.com.br

Recebido para publicação: 26/11/2007 - Aceito para publicação: 12/08/2008

\begin{abstract}
Resumo
A procura por espécies medicinais é crescente no Brasil, demandando, entre outras necessidades, pesquisas relacionadas à nutrição mineral de plantas. $\mathrm{O}$ objetivo foi avaliar o crescimento $\mathrm{e}$ caracterizar sintomas visuais de deficiência em mudas de espinheira-santa (Maytenus ilicifolia), em solução nutritiva sob condições de omissão de nutrientes. Os tratamentos foram omissão de N, P e K, solução completa e $\mathrm{H}_{2} \mathrm{O}$ desionizada. $\mathrm{O}$ experimento foi conduzido em casa de vegetação, utilizandose mudas de cinco meses de idade, mantidas por 105 dias em contato com solução nutritiva. As variáveis altura e diâmetro foram medidas mensalmente, para cálculo das taxas de crescimento. Avaliou-se a produção de matéria seca aérea e radicular, o volume radicular e os sintomas visuais de deficiência. O delineamento experimental foi em blocos ao acaso com cinco tratamentos e cinco repetições. A omissão de K não interferiu no crescimento em altura; a produção de matéria seca da parte aérea e da matéria seca total diminuiu com a omissão de $\mathrm{N}$; na omissão de $\mathrm{N}$ as plantas apresentaram clorose generalizada e baixo crescimento; na omissão de $\mathrm{P}$ ocorreu apenas menor expansão do limbo foliar; para o $\mathrm{K}$ verificou-se necrose em ponta e bordas das folhas mais velhas. Palavras-chave: Maytenus ilicifolia; sintomas visuais; nutrição de plantas.
\end{abstract}

\begin{abstract}
Growth and symptoms of nutritional deficiency in "espinheira-santa" seedlings with the omission of nitrogen, phosphorous and potassium. The demand for medicinal plants is growing in Brazil and this condition requires information about mineral nutrition of plants. The objective of this study was to characterize the symptoms of deficiency and growth in "espinheira-santa" (Maytenus ilicifolia) seedlings, cultivated under nutrient omission in nutrient solution. An experiment was conducted in greenhouse conditions, using the missing element technique. The seedlings were cultivated during 105 days in complete nutrient solution, with the omission of N, P, K from complete solution and only water. The variables plant height and diameter were measured monthly, and calculated the growth rate. At the end of the experiment was evaluated shoots and roots dry matter, volume of roots and the characteristics symptoms of nutrient deficiency. The experimental design was a completely randomized block with five treatments and five replications. Based on data analyzed the conclusions were the following: the omission of $\mathrm{K}$ did not affect the growth in height, the shoot and total dry matter were affected in the treatment without $\mathrm{N}$, under omission of $\mathrm{N}$ the plants showed chlorosis uniform and decrease growth; the omission of $\mathrm{P}$ demonstrated low leaf expansion and for $\mathrm{K}$, tip and marginal scorch necrosis on old leaves

Keywords: Maytenus ilicifolia; visual symptoms; plant nutrition.
\end{abstract}

\section{INTRODUÇÃO}

Atualmente, o interesse por plantas medicinais é impulsionado pelo desejo da população por uma vida mais saudável, assumindo crescente importância como recurso alternativo (homeopático), 
proporcionando um aumento considerável em sua procura (MARTINS et al., 1995). Essa espécie (Maytenus ilicifolia) no Brasil está ameaçada de extinção, por ser explorada em regime basicamente extrativista (BATALHA et al., 2002).

Maytenus ilicifolia Mart. ex Reiss é um subarbusto ou árvore ramificada desde a base e, em condições naturais, apresenta árvores adultas com altura média de 5,0 m. É conhecida popularmente por espinheira-santa (RS, SC), cancorosa (PR), espinheira-divina, erva cancrosa, erva-santa e cancerosa (RS). Pertence à ordem Celastrales e à família Celastraceae (CARVALHO-OKANO, 1992). De acordo com Negrelle; Leuchtenberger, (2001); Barddal (2002) e Radomski et al. (2004), a espécie ocorre em solos álicos, com baixa saturação em bases trocáveis, e em solos eutróficos. Esses solos frequentemente apresentam alto teor de matéria orgânica nos horizontes superficiais, indicativo importante para o desenvolvimento das práticas de adubação da espécie, principalmente em áreas de cultivo, o que supõe uma possível exigência de $\mathrm{N}$.

Os nutrientes minerais possuem funções essenciais e específicas no metabolismo das plantas, sendo que as espécies vegetais normalmente diferem na exigência nutricional, as quais devem ser conhecidas para uma melhor exploração. Assim, quando um dos nutrientes essenciais não está presente em quantidades satisfatórias às suas necessidades, ou em casos de combinações químicas que são pobremente absorvidas, essa deficiência gera anomalias devido a alterações no metabolismo vegetal (EPSTEIN e BLOOM, 2004). Esses distúrbios são característicos para cada nutriente, sendo relacionados às funções que este desempenha na planta.

O nitrogênio $(\mathrm{N})$ participa como constituinte de muitos componentes da célula vegetal, incluindo aminoácidos e ácidos nucleicos (TAIZ; ZEIGER, 2004). Conforme Raij (1991) e Tisdale et al. (1993), faz parte da molécula de clorofila e auxilia nas reações de síntese proteica. Esta, quando inibida, por consequência resulta na redução do processo de divisão celular (MALAVOLTA et al., 1989). O fósforo (P) está envolvido na transferência de energia, uma vez que a ATP (adenosina trifosfato) é necessária para a fotossíntese, para a translocação e outros processos metabólicos de relevância, esse elemento desempenha importante papel no metabolismo das plantas (SHUMAN, 1994). Segundo Taiz; Zeiger (2004), dentre as principais funções que o potássio (K) desempenha nas plantas, destacam-se a ativação enzimática, a participação ativa no estabelecimento do turgor celular e a manutenção da eletroneutralidade celular, além de estar envolvido na fotossíntese, no transporte de carboidratos, na síntese de proteínas, na expansão celular e no movimento estomático (MARSCHENER, 1995).

Uma das formas de se avaliar os problemas nutricionais é através do uso de soluções nutritivas, as quais são uma ferramenta rápida, principalmente, para a obtenção de informações sintomatológicas de carência e toxidez das plantas (NOVAIS et al., 1991; FURLANI et al., 1999; PARKER; NOVELL, 1999). Além desse conhecimento, informações sobre o crescimento tornam-se necessárias, de maneira a se aperfeiçoar o sistema de produção de mudas através de técnicas que aprimorem a qualidade das plantas. Segundo Carneiro (1995), a altura da parte aérea combinada com o diâmetro constitui um dos mais importantes parâmetros morfológicos para estimar o potencial de sobrevivência e de crescimento das mudas após o plantio definitivo no campo.

Atualmente existem poucos estudos relacionados à nutrição com espécies medicinais, principalmente com espinheira-santa. Dessa forma, este trabalho teve por objetivo avaliar o crescimento e sintomas visuais de deficiência em mudas de espinheira-santa (Maytenus ilicifolia), em solução nutritiva sob condições de omissão de N, P e K.

\section{MATERIAIS E MÉTODOS}

O experimento foi realizado em casa de vegetação do Departamento de Ciências do Solo da Universidade Federal do Paraná (UFPR), no período de agosto a dezembro de 2006.

As mudas de espinheira-santa foram produzidas em viveiro (empresa ervateira Baldo S.A., São Mateus do Sul, PR), a partir de sementes coletadas em janeiro de 2006 em matrizes locais, em substrato comercial para espécies nativas à base de casca de pinus e vermiculita. Cinco meses após a semeadura, elas tiveram as raízes lavadas e podadas a $6 \mathrm{~cm}$ de comprimento, sendo transferidas sem cotilédones para vasos com 2,0 $\mathrm{dm}^{3}$ de areia lavada $\left(\mathrm{pH}\right.$ de $5,7 \mathrm{em} \mathrm{CaCl}_{2} 0,01 \mathrm{~mol}^{-\mathrm{dm}^{-3}}$ ), colocados sobre suporte em contato com a solução (Figura 1B). 
Os tratamentos foram constituídos (Tabela 1) por: T1 = solução completa (testemunha); $\mathrm{T} 2=$ omissão de nitrogênio (-N); T3 = omissão de fósforo (-P); T4 = omissão de potássio (-K) e T5 = somente água deionizada $\left(\mathrm{H}_{2} \mathrm{O}\right)$. $\mathrm{O}$ experimento foi conduzido em blocos ao acaso com 5 repetições.

Inicialmente, utilizou-se solução (MOORE, 1974) diluída a 50\%, mantendo essa solução por duas semanas (fase de adaptação das mudas). Após esse período, ela foi substituída pela solução (Tabela 1) sem diluição (100\%), mantida até o final do experimento. As mudas foram irrigadas com a solução correspondente ao seu tratamento, conforme a necessidade, mantendo o nível da solução constante.

O crescimento das mudas foi avaliado aos $0,30,60$ e 90 dias, sendo a medida zero realizada após 15 dias do transplante. Mediu-se a altura (régua em $\mathrm{cm}$ ) e o diâmetro de colo (paquímetro em $\mathrm{mm}$, medido a $0,5 \mathrm{~cm}$ de altura em relação ao substrato), que foram também utilizados para a determinação das taxas mensais de crescimento em altura (TCA) e em diâmetro (TCD).

Tabela 1. Concentração da solução nutritiva correspondente a cada tratamento de Maytenus ilicifolia.

Table 1. Concentration of the corresponding nutritional solution for each treatment of Maytenus ilicifolia.

\begin{tabular}{|c|c|c|c|c|c|}
\hline \multirow{3}{*}{ Solução estoque } & \multicolumn{5}{|c|}{ Tratamentos } \\
\hline & Completa (T1) & $-\mathrm{N}(\mathrm{T} 2)$ & -P (T3) & -K (T4) & $\mathrm{H}_{2} \mathrm{O}$ (T5) \\
\hline & \multicolumn{5}{|c|}{$\ldots \ldots . . \mathbf{m l ~ d m}^{3} \ldots$} \\
\hline $\mathrm{Ca}\left(\mathrm{NO}_{3}\right)_{2} 1 \mathrm{M}$ & 05 & - & 05 & 05 & - \\
\hline $\mathrm{KNO}_{3} 1 \mathrm{M}$ & 05 & - & 05 & - & - \\
\hline $\mathrm{KH}_{2} \mathrm{PO}_{4} 1 \mathrm{M}$ & 01 & 01 & - & - & - \\
\hline $\mathrm{MgSO}_{4} 1 \mathrm{M}$ & 02 & 02 & 02 & 02 & - \\
\hline Fe EDTA & 01 & 01 & 01 & 01 & - \\
\hline Micro (-Fe) & 01 & 01 & 01 & 01 & - \\
\hline $\mathrm{KCl}$ & - & 05 & 01 & - & - \\
\hline $\mathrm{NaH}_{2} \mathrm{PO}_{4} 1 \mathrm{M}$ & - & 05 & - & 05 & - \\
\hline $\mathrm{NaNO}_{3} 1 \mathrm{M}$ & - & - & - & 05 & - \\
\hline
\end{tabular}

Decorridos 105 dias, caracterizaram-se os sintomas de deficiência nutricional, através da comparação visual entre os tratamentos com omissão de nutrientes e a solução completa. O volume de raízes (VR) foi medido por imersão em água. As amostras foram acondicionadas em sacos de papel e secas em estufa a $60{ }^{\circ} \mathrm{C}$ até peso constante. Determinaram-se a matéria seca da parte aérea (MSPA), a matéria seca da raiz (MSR) e a matéria seca total (MST). Em seguida, as amostras da parte aérea foram agrupadas por tratamento, moídas e analisadas quimicamente para os elementos $\mathrm{N}, \mathrm{P}, \mathrm{K}, \mathrm{Ca}, \mathrm{Mg}, \mathrm{Fe}, \mathrm{Mn}$, $\mathrm{Cu}$ e Zn, através dos métodos descritos por Martins e Reissmann (2007).

O delineamento experimental foi em blocos ao acaso, sendo os dados analisados estatisticamente através de ANOVA, pelo programa Assistat (SILVA e AZEVEDO, 2002), e as médias comparadas pelo Teste de Tukey ao nível de 5\% de probabilidade de erro.

\section{RESULTADOS E DISCUSSÃO}

\section{Crescimento das mudas}

Na primeira avaliação, nenhuma das variáveis apresentou diferença estatística significativa (Tabela 2), indicando que possivelmente as reservas presentes nas plantas foram suficientes para o seu crescimento durante esse período. Para o diâmetro médio, não houve diferença significativa até a terceira avaliação. Já para a altura, aos 30 dias os menores valores foram observados no tratamento $\mathrm{H}_{2} \mathrm{O}$, provavelmente causados pelo consumo das reservas nutricionais das mudas. Na terceira avaliação, aos 60 dias, as diferenças nas médias de crescimento médio em altura foram significativas, sendo os melhores resultados observados nos tratamentos com solução completa $(15,3 \mathrm{~cm}),-\mathrm{K}(13,84 \mathrm{~cm})$ e $-\mathrm{P}(12,10 \mathrm{~cm})$.

$\mathrm{Na}$ última avaliação (90 dias), os maiores valores de crescimento médio em altura foram constatados nos tratamentos com solução completa $(22,3 \mathrm{~cm})$ e $-\mathrm{K}(18,34 \mathrm{~cm})$. Já o tratamento $\mathrm{H}_{2} \mathrm{O}$ apresentou o menor crescimento em altura $(6,42 \mathrm{~cm})$ e diâmetro $(1,58 \mathrm{~mm})$. Observou-se que, dos 
nutrientes omitidos, os que mais afetaram negativamente o crescimento médio em altura foram $\mathrm{N}$ e $\mathrm{P}, \mathrm{o}$ que era esperado, uma vez que o $\mathrm{N}$ participa da estrutura de inúmeras moléculas, sendo o principal limitante do crescimento, pois faz parte de proteínas, ácidos nucleicos e muitos outros constituintes celulares, como membranas e hormônios (SOUZA; FERNANDES, 2007). Já para o P, segundo Malavolta et al. (1997), os efeitos mais marcantes de sua deficiência são a acentuada redução no crescimento como um todo. Venturin et al. (2005) relataram que a ausência desses mesmos nutrientes afetou drasticamente o crescimento das mudas de candeia (Eremanthus erythropappus), assim como Silva et al. (2005) observaram que as omissões dos nutrientes $\mathrm{N}$ e $\mathrm{P}$ foram os que mais limitaram o crescimento em altura e diâmetro das mudas de umbu (Spondias tuberosa), indicando que a M. ilicifolia é uma espécie sensível à ausência desses elementos.

Tabela 2. Média de dados de quatro avaliações de altura e diâmetro de Maytenus ilicifolia entre os períodos de setembro a dezembro de 2006 em casa de vegetação.

Table 2. Data average of four evaluations of height and diameter of Maytenus ilicifolia between the periods from september to december 2006 in greenhouse.

\begin{tabular}{|c|c|c|c|c|c|c|c|c|}
\hline \multirow{3}{*}{ Tratamento } & \multicolumn{8}{|c|}{ Avaliações (dias) } \\
\hline & $\mathbf{0}$ & 30 & 60 & 90 & $\mathbf{0}$ & 30 & 60 & 90 \\
\hline & \multicolumn{4}{|c|}{$\ldots \ldots \ldots \ldots \ldots$ Altura $\left(\mathrm{cm}\right.$ planta $\left.{ }^{-1}\right)}$. & \multicolumn{4}{|c|}{.... Diâmetro $\left(\mathrm{mm}_{\text {planta }}{ }^{-1}\right)$. } \\
\hline Completa & 5,60 & $8,87 \mathrm{a}$ & $15,3 \mathrm{a}$ & $22,30 \mathrm{a}$ & 1,09 & 1,46 & 1,86 & $2,28 \mathrm{a}$ \\
\hline$-\mathrm{N}$ & 5,44 & $7,40 \mathrm{ab}$ & $8,5 \mathrm{bc}$ & $8,94 \mathrm{~cd}$ & 1,06 & 1,45 & 1,67 & $1,86 a b$ \\
\hline$-\mathrm{P}$ & 5,84 & $9,04 \mathrm{a}$ & $12,10 \mathrm{ab}$ & $14,96 \mathrm{bc}$ & 1,04 & 1,30 & 1,65 & $1,90 \mathrm{ab}$ \\
\hline$-\mathrm{K}$ & 5,20 & $7,62 \mathrm{ab}$ & $13,84 \mathrm{a}$ & $18,34 \mathrm{ab}$ & 1,00 & 1,28 & 1,80 & $2,15 \mathrm{a}$ \\
\hline $\mathrm{H}_{2} \mathrm{O}$ & 4,36 & $6,00 \mathrm{~b}$ & $6,10 \mathrm{c}$ & $6,42 \mathrm{~d}$ & 0,90 & 1,23 & 1,43 & $1,58 \mathrm{~b}$ \\
\hline $\mathrm{CV}$ & $15,9 *$ & $14,3 * *$ & $24,4 * *$ & $24,5 * *$ & $21,7 *$ & $13,7 *$ & $15,47 *$ & $15,1 * *$ \\
\hline
\end{tabular}

*: Médias não diferem estatisticamente pelo teste de Tukey ao nível de 5\% de probabilidade de erro; **: Médias seguidas de letras diferentes diferem estatisticamente a $1 \%$.

Em relação à taxa de crescimento em altura (Tabela 3), diferenças estatísticas entre os tratamentos foram observadas a partir da segunda avaliação com valores inferiores nos tratamentos com $\mathrm{H}_{2} \mathrm{O}(0,10 \mathrm{~cm})$ e $-\mathrm{N}(1,10 \mathrm{~cm}) . \mathrm{Na}$ terceira avaliação, os tratamentos $\mathrm{H}_{2} \mathrm{O}(0,32 \mathrm{~cm})$ e $-\mathrm{N}(0,52 \mathrm{~cm})$ mantiveram-se apresentando as menores taxas. Medeiros e França (1986), em estudo com omissão de macronutrientes em algaroba (Prosopis juliflora), observaram que o $\mathrm{N}$ estava entre os nutrientes que mais afetaram o crescimento da planta, corroborando os resultados encontrados neste trabalho. Para a taxa de crescimento em diâmetro, não se observaram diferenças entre os tratamentos (Tabela 3).

Tabela 3. Média de dados de três avaliações da taxa de crescimento em altura e diâmetro em mudas de Maytenus ilicifolia.

Table 3. Data average of three evaluations of growth rate in height and diameter of Maytenus ilicifolia seedlings.

\begin{tabular}{|c|c|c|c|c|c|c|}
\hline \multirow[t]{2}{*}{ Tratamentos } & \multicolumn{3}{|c|}{$\begin{array}{c}\text { Taxa de crescimento em altura } \\
\left(\mathrm{cm} \text { planta }^{-1}\right)\end{array}$} & \multicolumn{3}{|c|}{$\begin{array}{l}\text { Taxa de crescimento em diâmetro } \\
\left(\text { mm planta }^{-1}\right)\end{array}$} \\
\hline & $1^{\circ}$ out/set & $2^{\circ}$ nov/out & $3^{\circ} \mathrm{dez} / \mathrm{nov}$ & $1^{\circ}$ out/set & $2^{\circ}$ nov/out & $3^{\circ} \mathrm{dez} / \mathrm{nov}$ \\
\hline Completa & 3,3 & $6,4 \mathrm{a}$ & $7,0 \mathrm{a}$ & 0,37 & 0,39 & 0,41 \\
\hline$-\mathrm{N}$ & 2,0 & $1,1 \mathrm{~b}$ & $0,5 \mathrm{c}$ & 0,39 & 0,22 & 0,19 \\
\hline$-P$ & 3,2 & $3,0 \mathrm{ab}$ & $2,9 \mathrm{~b}$ & 0,25 & 0,35 & 0,25 \\
\hline$-K$ & 2,4 & $6,2 \mathrm{a}$ & $4,5 \mathrm{~b}$ & 0,28 & 0,51 & 0,35 \\
\hline $\mathrm{H}_{2} \mathrm{O}$ & 1,6 & $0,4 \mathrm{~b}$ & $0,3 \mathrm{c}$ & 0,32 & 0,22 & 0,15 \\
\hline $\mathrm{CV}$ & $48,5^{*}$ & $57,1^{* *}$ & $38,1^{* *}$ & $38,9^{*}$ & $66,3^{*}$ & $58,1 *$ \\
\hline
\end{tabular}

*: Médias não diferem estatisticamente pelo teste de Tukey ao nível de 5\% de probabilidade de erro; **: Médias seguidas de letras diferentes diferem estatisticamente a $1 \%$. 
Os melhores tratamentos para a variável matéria seca da parte aérea e radicular foram soluções completa e -K (Tabela 4). Em relação à matéria seca radicular, à matéria seca total e ao volume radicular, o tratamento que mais afetou negativamente esses parâmetros foi o $\mathrm{H}_{2} \mathrm{O}$ (Tabela 4).

$\mathrm{O}$ estudo demonstrou que a falta de $\mathrm{N}$ foi determinante na redução do peso da matéria seca da parte aérea, devido suas importantes funções nas plantas, já demonstradas anteriormente. Bellote; Sturion (1985), em estudo com omissões de nutrientes em hidroponia, verificaram que o $\mathrm{N}$ foi o elemento mais limitante à produção de matéria seca em mudas de erva-mate, vindo em seguida, em ordem decrescente, $\mathrm{Ca}, \mathrm{P}, \mathrm{K}, \mathrm{Mg}, \mathrm{Zn}, \mathrm{Cu}$ e Fe. Resultados semelhantes foram encontrados por Dias et al. (1994) trabalhando com omissões de nutrientes em acácia. Observaram que no tratamento com omissão de $\mathrm{N}$ houve uma redução do peso seco da parte aérea e menor acúmulo de peso seco total.

Verificou-se que, de maneira geral a omissão de N, P e K reduziu a relação MSPA/MSR. Segundo Zonta et al. (2006) e Taiz; Zeiger (2004), plantas em condições de deficiências nutricionais tendem a investir mais energia na produção de raízes para ampliar o potencial de absorção desses nutrientes. $\mathrm{ON}$, por ser normalmente o elemento mais requerido pela maioria das plantas (Souza e Fernandes, 2006), neste trabalho, em condição de omissão, $\mathrm{N}$ foi o elemento que mais afetou essa variável.

A superioridade da relação parte aérea/parte radicular (Tabela 4) para a solução completa, especialmente para o $\mathrm{N}$, pode ser em virtude de as plantas com déficit de nutrientes terem desenvolvido mecanismos de sobrevivência: em condições de recursos nutricionais escassos, maior é o investimento no sistema radicular, porém, quando em extrema deficiência, podem diminuir ou até mesmo cessar o crescimento radicular. À medida que a disponibilidade de nutrientes melhora, o crescimento radicular aumenta, sendo que em condições de abundância de nutrientes o crescimento radicular pode diminuir, pois um pequeno volume de raízes é suficiente para suprir a planta. Essa hipótese é reforçada por Zonta et al. (2006) e Taiz ; Zeiger (2004).

Tabela 4. Média de dados para massa seca da parte aérea (MSPA), massa seca radicular (MSR), massa seca total (MST), relação massa seca parte aérea com massa seca radicular (MSPA/MSR) e volume radicular (VR) em mudas de Maytenus ilicifolia.

Table 4. Data average of the aerial part (MSPA), dry mass roots dry mass (MSR), total dry mass (MST), relationship between aerial part and root mass (MSPA/MSR) and volume of roots (VR) in seedlings of de Maytenus ilicifolia.

\begin{tabular}{|c|c|c|c|c|c|}
\hline \multirow{2}{*}{ Tratamento } & MSPA & MSR & MST & \multirow{2}{*}{ MSPA/MSR } & \multirow{2}{*}{$\frac{\text { VR }}{\mathrm{cm}^{3} \text { planta }}$} \\
\hline & \multicolumn{3}{|c|}{$\ldots$} & & \\
\hline Completa & $1,50 \mathrm{a}$ & $0,22 \mathrm{a}$ & $1,72 \mathrm{a}$ & $7,1 \mathrm{a}$ & $1,00 \mathrm{a}$ \\
\hline$-\mathrm{N}$ & $0,45 \mathrm{c}$ & $0,16 \mathrm{ab}$ & $0,61 \mathrm{~cd}$ & $3,32 \mathrm{~b}$ & $0,85 \mathrm{a}$ \\
\hline$-\mathrm{P}$ & $0,86 \mathrm{bc}$ & $0,17 \mathrm{ab}$ & $1,04 \mathrm{bc}$ & $4,96 \mathrm{ab}$ & $0,99 \mathrm{a}$ \\
\hline$-K$ & $1,12 \mathrm{a}$ & $0,21 \mathrm{a}$ & $1,33 \mathrm{ab}$ & $5,56 \mathrm{ab}$ & $0,82 \mathrm{ab}$ \\
\hline $\mathrm{H}_{2} \mathrm{O}$ & $0,4 \mathrm{c}$ & $0,10 \mathrm{~b}$ & $0,5 \mathrm{~d}$ & $3,96 \mathrm{ab}$ & $0,51 \mathrm{~b}$ \\
\hline $\mathrm{CV}$ & $29,1 * *$ & $31,8^{* *}$ & $25,6 * *$ & $35,4 * *$ & $19,1 * *$ \\
\hline
\end{tabular}

**: Médias seguidas de letras diferentes diferem estatisticamente a $1 \%$.

\section{Sintomas visuais de deficiência e teores de nutrientes}

De maneira geral, verificaram-se sintomas visuais de deficiência muito semelhantes para os tratamentos $-\mathrm{N}$ e $\mathrm{H}_{2} \mathrm{O}$ (Figura 1A), embora a clorose tenha apresentado maior expressão no tratamento $\mathrm{H}_{2} \mathrm{O}$ (Figura $1 \mathrm{G}$ ).

Acompanhando o desenvolvimento das mudas de espinheira-santa submetidas à solução nutritiva com omissão de $\mathrm{N}$, no decorrer do experimento observou-se que as plantas demonstraram um desenvolvimento abaixo do normal, com clorose generalizada (Figura 1D), o que era esperado devido às funções básicas exercidas pelo $\mathrm{N}$ nas plantas, já citadas por diversos autores (MARSCHENER, 1995; MENGEL; KIRKBY, 1982). Esse mesmo sintoma foi observado por Maffeis et al. (2000) em Eucalyptus citriodora em condição de omissão de $\mathrm{N}$.

$\mathrm{Na}$ ausência de $\mathrm{P}$, os sintomas foram pouco relevantes, observando-se apenas uma menor expansão do limbo foliar. De acordo com Malavolta et al. (1997), um dos efeitos mais marcantes da deficiência de P é a acentuada redução no crescimento como um todo, porém para a espinheira-santa esse 
efeito não foi imediato (Tabela 2). Segundo Raij (1991), os sintomas de deficiência de P não são expressivos como os de $\mathrm{N}$ e $\mathrm{K}$. A não detecção de sintomas visuais pode ser devida à utilização do $\mathrm{P}$ de reserva da semente sob a forma de fitatos, que somam $50 \%$ a $80 \%$ do fósforo total nas sementes (LOTT et al., 1995), que poderia ter suprido de $\mathrm{P}$ a planta no início de seu desenvolvimento.

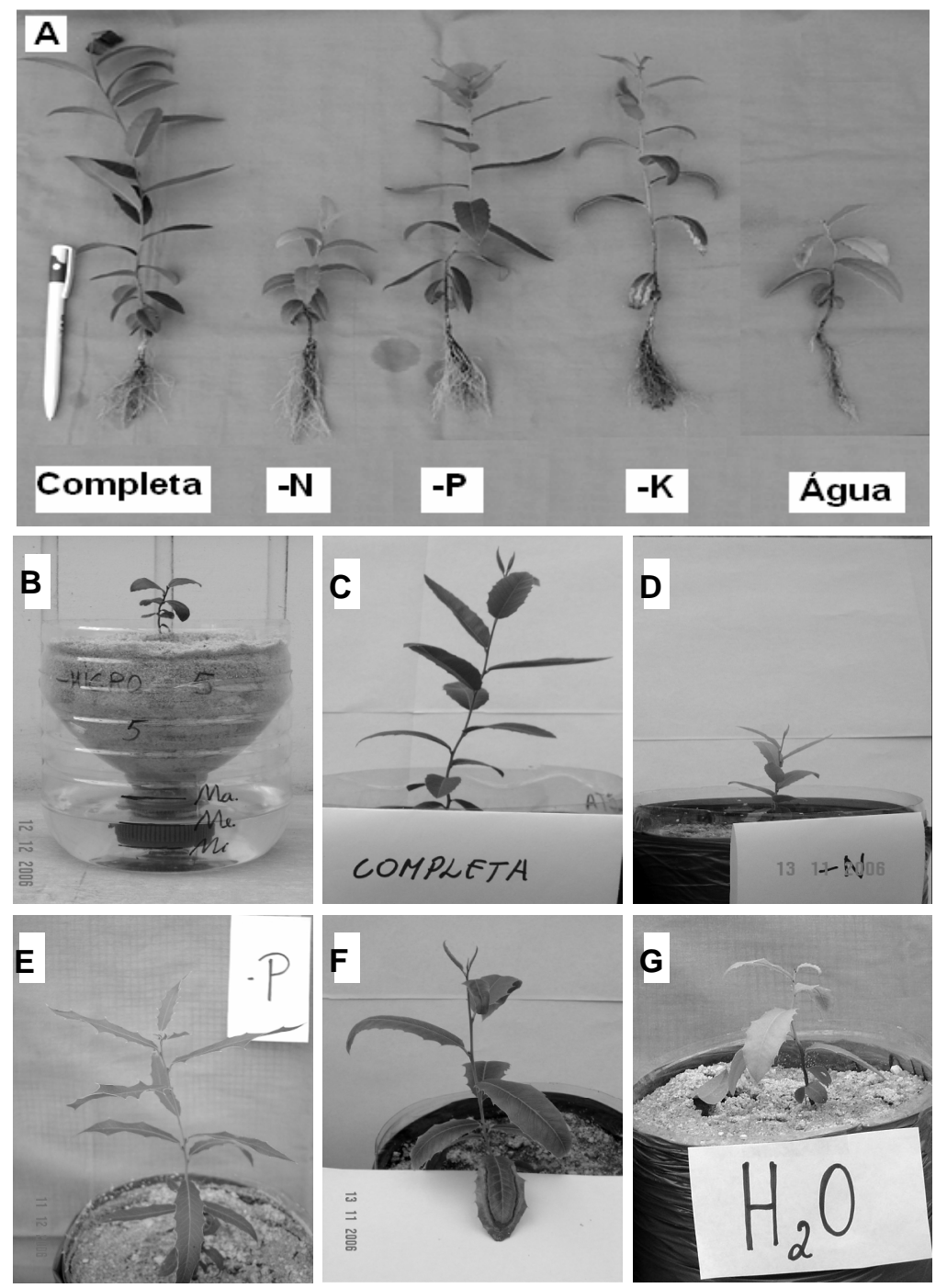

Figura 1. Mudas de Maytenus ilicifolia após 105 dias em soluções nutritivas. (A) Visualização geral de uma planta representativa de cada tratamento, (B) vaso e suporte com solução, (C) tratamento solução completa; (D) tratamento -N, (E); tratamento -P, (F); tratamento - K e (G) tratamento $\mathrm{H}_{2} \mathrm{O}$.

Figure 1. Seedlings of Maytenus ilicifolia after 105 days in nutrient solution. (A) General view of a representative of each treatment plant, (B) support vessel with solution, (C) treatment complete solution, (D) treatment-N, (E); treatment-P, (F); treatment - K e (G) treatment $\mathrm{H}_{2} \mathrm{O}$.

$\mathrm{O}$ tratamento com omissão de $\mathrm{K}$ apresentou sintomas característicos de deficiência desse elemento, com necrose em ponta e bordas das folhas mais velhas (Figura 1F). Esses sintomas são típicos na literatura, possivelmente provocados pelo acúmulo de putrescina, uma vez que esta, segundo Marschener (1995), é produzida na deficiência de K. Medeiros; França (1986), estudando a omissão de macronutrientes em algaroba (Prosopis juliflora), observaram sintomas semelhantes na ausência de K, corroborando os resultados aqui apresentados. 
Os valores médios encontrados de macro e micronutrientes em mudas de M. ilicifolia estão apresentados na tabela 5. Verifica-se que os teores $\left(\mathrm{g} \cdot \mathrm{kg}^{-1}\right)$ dos macronutrientes nas folhas do tratamento completo e com omissão dos nutrientes foram, respectivamente, $\mathrm{N}=18,0$ e 7,9, $\mathrm{P}=3,8$ e 0,9 e $\mathrm{K}=18,7$ e 4,3. De acordo com a análise foliar para a solução completa, a ordem decrescente dos nutrientes analisados nas folhas foi: $\mathrm{K}>\mathrm{N}>\mathrm{Ca}>\mathrm{P}>\mathrm{Mg}>\mathrm{Fe}>\mathrm{Mn}>\mathrm{Zn}>\mathrm{Cu}$. Tal resultado diferiu dos de Radomski (1998), que obteve $\mathrm{K}>\mathrm{N}>\mathrm{Ca}>\mathrm{Mg}>\mathrm{P}>\mathrm{Mn}>\mathrm{Fe}>\mathrm{Zn}>\mathrm{Cu}$, provavelmente devido ao seu trabalho ter sido em diferentes tipos de solo, com plantas adultas, com teores foliares de $\mathrm{N}, \mathrm{P}, \mathrm{K}, \mathrm{Ca}$ e $\mathrm{Mg}$ iguais a 16,$7 ; 15,9 ; 9,9 ; 3,8$ e $2,7\left(\mathrm{~g} \mathrm{~kg}^{-1}\right)$, respectivamente.

Tabela 5. Teores de macro e micronutrientes de tecidos de Maytenus ilicifolia, dezembro de 2006, em casa de vegetação.

Table 5. Levels of macro and micronutrients of tissue of Maytenus ilicifolia, December 2006 in greenhouse.

\begin{tabular}{|c|c|c|c|c|c|c|c|c|c|}
\hline \multirow{2}{*}{ Tratamentos } & $\mathbf{N}$ & $\mathbf{P}$ & $\mathbf{K}$ & $\mathbf{C a}$ & Mg & $\mathbf{C u}$ & $\mathrm{Fe}$ & Mn & Zn \\
\hline & \multicolumn{5}{|c|}{ 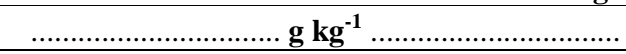 } & \multicolumn{4}{|c|}{$\ldots \ldots \ldots \ldots \ldots \ldots \ldots . . \mathrm{mg} \mathrm{kg}^{-1} \ldots \ldots \ldots \ldots \ldots \ldots \ldots$} \\
\hline Completa & 18,0 & 3,8 & 18,7 & 6,6 & 2,2 & 0,30 & 418 & 19 & 6,0 \\
\hline$-\mathrm{N}$ & 7,9 & 3,9 & 27,9 & 6,2 & 2,4 & 0,60 & 403 & 80 & 8,0 \\
\hline$-\mathrm{P}$ & 17,4 & 0,9 & 21,4 & 9,9 & 1,6 & 0,50 & 377 & 28 & 7,0 \\
\hline$-K$ & 16,9 & 10,3 & 4,3 & 6,0 & 2,5 & 0,03 & 374 & 32 & 7,0 \\
\hline Água & 8,7 & 1,5 & 7,7 & 3,6 & 1,1 & 0,03 & 402 & 63 & 0,0 \\
\hline
\end{tabular}

Considerando o bom desenvolvimento das mudas do tratamento completo para todas as variáveis de crescimento avaliadas e o bom aspecto visual dessas mudas, tais resultados de composição da parte aérea, embora sem análise estatística devido à quantidade de material disponível para a análise, poderão servir como referencial para a avaliação do estado nutricional de mudas de espinheira-santa.

\section{CONCLUSÕES}

- A ausência de K não afetou o crescimento em altura.

- As ausências de $\mathrm{N}$ e $\mathrm{P}$ foram as que mais afetaram a taxa de crescimento em altura e a produção de matéria seca da parte aérea e total.

- $\quad \mathrm{Na}$ omissão de $\mathrm{N}$, as plantas apresentaram clorose generalizada e baixo crescimento.

- Na omissão de P, ocorreu apenas menor expansão do limbo foliar.

- $\mathrm{Na}$ omissão de $\mathrm{K}$, verificou-se necrose em ponta e bordas das folhas mais velhas.

\section{REFERÊNCIAS}

BARDDAL, M. L. Aspectos florísticos e fitossociológicos do componente arbóreo-arbustivo de uma Floresta Ombrófila Mista Aluvial - Araucária, PR. 89 f. Dissertação (Mestrado em Ciências Florestais) - Setor de Ciências Agrárias, Universidade Federal do Paraná, Curitiba, 2002.

BATAlHA, M. O.; MING, L. C.; NANTES, J. F. D.; ALCÂNTARA, R. C. L.; CASTRO, D. M.; MACHADO, J. G.; LOURENZANI, A. E. B.; BARRETO, C. V. Complexo agroindustrial de plantas medicinais e aromáticas no Estado de São Paulo: Diagnóstico e proposição de ações e melhoria da eficiência e da competitividade. Sumário Executivo do projeto. São Carlos: UFSCAR/ UNESP/ SEBRAE, 2002. 57p.

BELlOTE, A. F. J.; STURION, J. A. Deficiências minerais em erva-mate (Ilex paraguariensis St. Hil.) Resultados preliminares. In: SEMINÁRIO SOBRE ATUALIDADES E PERSPECTIVAS FLORESTAIS (Silvicultura da erva-mate (Ilex paraguariensis St. Hil)), 10. 1985, Curitiba. Anais... Curitiba: EMBRAPA-CNPF, 1985, p. 124-127.

CARNEIRO, J. G. de A. Produção e controle de qualidade de mudas florestais. Curitiba: UFPR/FUPEF, Campos: UENF, 1995. 451 p. 
CARVAlHO-OKANO, R. M. de. Estudos taxonômicos do gênero Maytenus Mol. Emend. Mol. (Celastraceae) do Brasil extra-amazônico. 257 p. Tese (Doutorado em Ciências - Biologia Vegetal) Instituto de Biologia da Universidade Estadual de Campinas, Campinas, 1992.

DIAS, L. E.; FARIAS, S. M.; FRANCO, A. A. Crescimento de mudas de Acacia mangium Willd em resposta à omissão de macronutrientes. Revista Árvore, Viçosa, MG, v. 18, p. 123-131. 1994.

EPSTEIN, E.; BLOOM, A. J. Nutrição mineral de plantas: princípios e perspectivas. 2. ed. Londrina: Ed. Plantas, 2004. 403 p.

FURLANI, P. R.; SILVEIRA, L. C. P.; BOLONHEZI, D.; FANQUIN, V. Cultivo hidropônico de plantas. Campinas: Instituto Agronômico, 1999. 52 p. (Boletim Técnico 180).

LOTT, J. N.; GREENWOOD, J. S.; BATTEM, G. D. Mechanisms and regulation of mineral nutrient storage during seed development. In: KIGEL, J.; GALILI. Seed Development and Germination., New York: M. Dekker, 1995. p. 215-235.

MAFFEIS, A. R.; SILVEIRA, R. L. V. A; BRITO, J. O. Reflexo das deficiências de macronutrientes e boro no crescimento de planta, produção e qualidade de óleo essencial em Eucalyptus citriodora. Scientia Forestalis, Piracicaba, n. 57, p. 87-98, 2000.

MALAVOLTA, E.; VITTI, G. C.; OLIVEIRA, S. A. Avaliação do estado nutricional das plantas: princípios aplicações. 2 ed. Piracicaba; POTAFOS, 1989. 201 p.

MARSCHENER, H. Mineral nutrition of higher plants. 2. ed. London: Academic, 1995. 889 p.

MARTINS, A. P. L.; REISSMANN, C. B. Material vegetal e as rotinas laboratoriais nos procedimentos químico-analíticos. Curitiba. Scientia Agraria, Curitiba, v. 8, n. 1, p. 1-17, 2007.

MARTINS, E. R.; CASTRO, D. M.; CASTELLANI, D. C.; DIAS, J. E. Plantas medicinais. Viçosa MG: UFV, 1995. $220 \mathrm{p}$.

MEDEIROS, A. A. de; FRANÇA, A. F. de S. Desnutrição de macronutrientes em plantas de algaroba. IPEF, Piracicaba, n. 32, p. 53-55, 1986.

MENGEL, K.; KIRKBY, E. A. Principles of plant nutrition. 3 ed. Bern: International Potash Institute. $1982,655 \mathrm{p}$.

MOORE, T. C. Research experiences in plant physiology: a laboratory manual. New Yor: SpringerVerlag, 1974. $462 \mathrm{p}$.

NEGRELLE, R. R. B.; LEUCHTENBERGER, R. Composição e estrutura do componente arbóreo de um remanescente de floresta ombrófila mista. Floresta, Curitiba, v. 31, 2001.

NOVAIS, R. F.; NEVES, J. C. L.; BARROS, N. F. Ensaio em ambiente controlado. In: OLIVEIRA, A. J.; Garrido, W. E.; Araújo, J. D.; Lourenço, S. (Eds.). Métodos de pesquisa em fertilidade do solo. Brasília, DF: EMBRAPA - SEA, 1991. p. 189-255.

PARKER, D. R.; NORVEL. W. A. Advances in solution culture methods for plant mineral nutrition research. In: SPARKS, D. L. (Ed.). Advances in Agronomy. New York: Academic Press, 1999. v. 65, p. 151-213.

RADOMSKI, M. I. Caracterização ecológica e fitoquímica de Maytenus ilicifolia Mart., em populações nativas, no município da Lapa - Paraná. 97 f. Dissertação (Mestrado em Ciência do Solo) - Setor de Ciências Agrárias, Universidade Federal do Paraná, Curitiba, 1998.

RADOMSKI, M. I.; WISNIEWSKI, C.; CURCIO, G. R.; RACHWAL, M. G.; SANTOS, C. A. M. Caracterização de ambientes de ocorrência natural e sua influência sobre o peso específico e o teor de polifenóis totais de folhas de espinheira-santa (Maytenus ilicifolia. Mart.). Revista Brasileira de Plantas Medicinais, Botucatu, v. 6, n. 2, p. 65-68, 2004.

RAIJ, B. V. Fertilidade do solo e adubação. Piracicaba: CERES/POTAFÓS, 1991, 343 p. 
REISSMANN, C. B.; CARNEIRO, C. Crescimento e composição química da erva-mate (Ilex paraguariensis St. Hil.), transcorrido oito anos de calagem. Floresta, Curitiba, v. 34, n. 3, p. 281-386, 2004.

SCHUMAN, L. M. Mineral Nutrition. In: WILKINSON, R. E. (Ed). Plant-environment interactions. New York: M. Dekker, 1994. p. 149-182.

SILVA, E. B.; GONÇALVES, N. P.; PINHO, P. J. Limitações nutricionais para crescimento de mudas de umbuzeiro em Latossolo Vermelho distrófico no Norte de Minas. Acta Scientiarum Agronomy, Maringá, v. 27, n. 1, p. 55-59, 2005.

SILVA, F. A. S.; AZEVEDO, C. A. V. de. Versão do programa computacional Assistat para o sistema operacional Windows. Revista Brasileira de Produtos Agroindustriais, Campina Grande, v. 4, n. 1, p. 71-78, 2002.

SOUZA, S. R.; FERNANDES, M. S. Nitrogênio. In: FERNANDES, M. S. Nutrição Mineral de Plantas. Viçosa, MG: Sociedade Brasileira de Ciência do Solo, 2006. p. 215-252.

TAIZ, L.; ZEIGER, E. Fisiologia vegetal. 3. ed. Porto Alegre: Artmed, 2004. 719 p.

TISDALE, S. L.; NELSON, W. L.; BEATON, J. D.; HAVLIN, J. L. Soil and fertilizer nitrogen. In: TISDALE, S. L.; NELSON, W. L.; BEATON, J. D.; HAVLIN, J. L. (Eds.). Soil fertility and fertilizer. New York: Macmillan, 1993. p. 109-175.

VENTURIN, N.; SOUZA, P. A.; MACEDO, R. L. G. de; NOGUEIRA, F. D. Adubação mineral da candeia (Eremanthus erythropapus (DC.) Mcleish). Revista Floresta, Curitiba, v. 35, n. 2, p. 211-219, 2005.

ZONTA, E.; BRASIL, F. C.; GOI, S. R.; ROSA, M. M. T. O sistema radicular e suas interações com o ambiente edáfico. In: FERNANDES, M. S. Nutrição mineral de plantas. Viçosa, MG: Sociedade Brasileira de Ciência do Solo, 2006. p. 07-52. 